\title{
Asymptotic properties of a population model with Allee effects in random environments
}

\author{
Famei Zheng ${ }^{1}$ and Guixin $\mathrm{Hu}^{2 *}$
}

\section{"Correspondence:}

huzhang2009hao@163.com

${ }^{2}$ School of Mathematics and Information Science, Henan

Polytechnic University, Jiaozuo, 454000, P.R. China

Full list of author information is available at the end of the article

\begin{abstract}
In this research, we probe the influences of two common types of stochastic noises in the environment, namely, white noise and telephone noise, and put forward a stochastic differential equation population model with Allee effects. We analyze some asymptotic behaviors of the model, including extermination, persistence and invariant measure. Some vital functions of white noise and telephone noise on these asymptotic behaviors of the model are discovered and numerically expounded.
\end{abstract}

MSC: $60 \mathrm{H} 10 ; 60 \mathrm{H} 30 ; 92 \mathrm{D} 25$

Keywords: Allee effect; Environmental noises; Stability; Persistence

\section{Introduction}

In the nature, species often forms various degrees of aggregations [17]. Aggregation may enhance intraspecific competition, but this influence is often offset by the increased survival ability of the species, because aggregation could help the individual to protect itself, discover resources, or adapt to the environmental stresses [17]. The degree of aggregation, which varies with species and circumstances, promotes optimum population growth and survival. A group often has an optimal population size at some intermediate level. Both undercrowding and overcrowding have limitations [16]. This aggregation principle is the famous Allee principle [16].

To mathematically depict the Allee principle, several models have been put forward (see, e.g., $[4,5,8])$. Especially, Jacobs [5] has put forward the following deterministic differential equation model and probed its stability:

$$
\frac{\mathrm{d} N(t)}{\mathrm{d} t}=N(t)\left[r+\frac{\rho N^{a}(t)}{N^{a}(t)+\theta}-\beta N^{b}(t)\right]
$$

where $N(t)$ is the population abundance; $r>0, \rho>0$ and $\beta>0$ measure the growth rate, the positive influence of aggregation and the negative influence of overcrowding, respectively. $a>0$ and $b>0$ are flexible constants to fit the real data.

Nevertheless, environmental noises should be thought over owing to their universal existence [15]. In fact, ignoring environmental noises may be one of the main causes for the

(c) The Author(s) 2021. This article is licensed under a Creative Commons Attribution 4.0 International License, which permits use, sharing, adaptation, distribution and reproduction in any medium or format, as long as you give appropriate credit to the original author(s) and the source, provide a link to the Creative Commons licence, and indicate if changes were made. The images or other third party material in this article are included in the article's Creative Commons licence, unless indicated otherwise in a credit line to the material. If material is not included in the article's Creative Commons licence and your intended use is not permitted by statutory regulation or exceeds the permitted use, you will need to obtain permission directly from the copyright holder. To view a copy of this licence, visit http://creativecommons.org/licenses/by/4.0/. 
vanishing of many species [10]. As a result, one should concentrate on model (1) with environmental noises to exploit the Allee principle more accurately. However, no findings of model (1) with environmental noises have been reported.

The goal of this research is to put forward a stochastic form of model (1) and to probe its dynamical behaviors. We put forward the stochastic differential equation model in Sect. 2. Then we probe the extermination-persistence and invariant measure of the model in Sects. 3 and 4, respectively. Afterwards, we discuss and numerically expound the impacts of environmental noises on the properties of model (3) in Sect. 5. Finally, we summarize the research in Sect. 6.

\section{The stochastic model}

Two common types of stochastic noises in the environment are white noise and telephone noise [19]. White noise could influence one or more parameters in a population model [15]; and the growth rate might be the most sensitive parameter [2]. There are several means to bring white noise to the growth rate. One widely adopted means (see, e.g., [6, 7, $13])$ is to presume that

$$
r \rightarrow r+\varpi \dot{\omega}(t)
$$

where $\dot{\omega}(t)$ is white noise, namely, $\{\omega(t)\}_{t \geq 0}$ is a standard Brownian motion, $\varpi^{2}$ represents the intensity. Therefore, model (1) turns into

$$
\mathrm{d} N(t)=N(t)\left[r+\frac{\rho N^{a}(t)}{N^{a}(t)+\theta}-\beta N^{b}(t)\right] \mathrm{d} t+\varpi N(t) \mathrm{d} \omega(t) .
$$

Now we move to telephone noise which can cause the changes of parameters [12]. Mathematically, one can recur to a right continuous irreducible Markov chain $\kappa(t)$ with a finite state space $\Upsilon=\left\{1, \ldots, N^{*}\right\}$ to describe telephone noise [20]. Adhering to this means, model (2) turns into

$$
\mathrm{d} N(t)=N(t)\left[r(\kappa(t))+\frac{\rho(\kappa(t)) N^{a}(t)}{N^{a}(t)+\theta(\kappa(t))}-\beta(\kappa(t)) N^{b}(t)\right] \mathrm{d} t+\varpi(\kappa(t)) N(t) \mathrm{d} \omega(t),
$$

where $\min _{i \in \Upsilon}\{r(i), \rho(i), \theta(i)\}>0$. Model (3) could depict the reality better than model (2). As a matter of fact, assume initially, $\kappa(t)=i_{0}$, then model (3) is as follows:

$$
\mathrm{d} N(t)=N(t)\left[r\left(i_{0}\right)+\frac{\rho\left(i_{0}\right) N^{a}(t)}{N^{a}(t)+\theta\left(i_{0}\right)}-\beta\left(i_{0}\right) N^{b}(t)\right] \mathrm{d} t+\varpi\left(i_{0}\right) N(t) \mathrm{d} \omega(t)
$$

for a random time, until $\kappa(t)$ jumps to a new state, $i_{1}$, then model (3) is as follows:

$$
\mathrm{d} N(t)=N(t)\left[r\left(i_{1}\right)+\frac{\rho\left(i_{1}\right) N^{a}(t)}{N^{a}(t)+\theta\left(i_{1}\right)}-\beta\left(i_{1}\right) N^{b}(t)\right] \mathrm{d} t+\varpi\left(i_{1}\right) N(t) \mathrm{d} \omega(t)
$$

until $\kappa(t)$ jumps again. Clearly, if $\kappa(t)$ does not jump, then model (3) reduces to model (2). As a result, model (3) could depict the random changes of the environment, which cannot be described by model (2). 


\section{Extinction and persistence}

Let $\kappa(t)$ be independent with $\omega(t)$, and let $M=\left(m_{i l}\right)_{N^{*} \times N^{*}}$ and $\phi=\left(\phi_{1}, \ldots, \phi_{N^{*}}\right)$ represent the generator and the invariant measure of $\kappa(t)$, respectively. Then, for $i \neq l, m_{i l}>0$, and, for $i \in \Upsilon, \sum_{l \in \Upsilon} m_{i l}=0$. Define

$$
\varsigma^{u}=\max _{i \in \Upsilon}\{\varsigma(i)\}, \quad \varsigma^{l}=\min _{i \in \Upsilon}\{\varsigma(i)\} .
$$

For the scalar equation

$$
\mathrm{d} z(t)=f_{1}(z(t), \kappa(t)) \mathrm{d} t+f_{2}(z(t), \kappa(t)) \mathrm{d} \omega(t)
$$

define an operator $\mathcal{L}$ as follows:

$$
\mathcal{L} \Psi(z, i)=\Psi_{z}(z, i) f_{1}(z, i)+f_{2}^{2}(z, i) \Psi_{z z}(z, i) / 2+\sum_{l \in \Upsilon} m_{i l} \Psi(z, l)
$$

where $\Psi(z, i)$ is a twice differentiable function of $z$.

Lemma 1 ([9], Lemma 2.3) Let $v \in \mathbb{R}^{N^{*}}$ be a vector, then $\phi v=0$ means that $M x=v$ possesses a solution.

Lemma 2 For arbitrary $(N(0), \kappa(0)) \in(0,+\infty) \times \Upsilon$, model (3) has a unique solution $(N(t), \kappa(t)) \in(0,+\infty) \times \Upsilon$ for all $t \geq 0$ a.s.

Proof The proof is standard and therefore is omitted (see, e.g., [19]).

Now, let us probe the extermination of (3).

Theorem 1 If $\bar{\Delta}+\bar{\rho}<0$, then $\lim _{t \rightarrow+\infty} N(t)=0$, a.s., here

$$
\bar{\Delta}=\sum_{i \in \Upsilon} \phi_{i} \Delta(i), \quad \Delta(i)=r(i)-\varpi^{2}(i) / 2, \quad \bar{\rho}=\sum_{i \in \Upsilon} \phi_{i} \rho(i) .
$$

Proof According to Itô's formula [14],

$$
\begin{aligned}
& \ln N(t)-\ln N(0) \\
& \quad=\int_{0}^{t}\left[\Delta(\kappa(s))+\rho(\kappa(s)) \frac{N^{a}(s)}{N^{a}(s)+\theta(\kappa(s))}-\beta(\kappa(s)) N^{b}(s)\right] \mathrm{d} s+\int_{0}^{t} \varpi(\kappa(s)) \mathrm{d} \omega(s) \\
& \quad \leq \int_{0}^{t}[\Delta(\kappa(s))+\rho(\kappa(s))] \mathrm{d} s+\int_{0}^{t} \varpi(\kappa(s)) \mathrm{d} \omega(s) .
\end{aligned}
$$

Taking advantage of the ergodicity of $\phi$ leads to

$$
\lim _{t \rightarrow+\infty} t^{-1} \int_{0}^{t}[\Delta(\kappa(s))+\rho(\kappa(s))] \mathrm{d} s=\bar{\Delta}+\bar{\rho} .
$$


The strong number law of martingales [14] implies

$$
\lim _{t \rightarrow+\infty} t^{-1} \int_{0}^{t} \varpi(\kappa(s)) \mathrm{d} \omega(s)=0, \quad \text { a.s. }
$$

By (4), (5) and (6),

$$
\limsup _{t \rightarrow+\infty} t^{-1} \ln N(t) \leq \bar{\Delta}+\bar{\rho}<0, \quad \text { a.s. }
$$

So $\lim _{t \rightarrow+\infty} N(t)=0$, a.s.

Theorem 2 If $\bar{\Delta}>0$, then the species represented by Eq. (3) is stochastically persistent, namely, for any $\varepsilon \in(0,1)$, one can seek a pair of constants $\Lambda_{1}=\Lambda_{1}(\varepsilon) \in(0,+\infty)$ and $\Lambda_{2}=$ $\Lambda_{2}(\varepsilon) \in(0,+\infty)$ such that, for arbitrary $(N(0), \kappa(0)) \in(0,+\infty) \times \Upsilon$,

$$
\liminf _{t \rightarrow+\infty} \mathrm{P}\left\{N(t) \leq \Lambda_{1}\right\} \geq 1-\varepsilon
$$

and

$$
\liminf _{t \rightarrow+\infty} \mathrm{P}\left\{N(t) \geq \Lambda_{2}\right\} \geq 1-\varepsilon
$$

Proof We address $(7)$ to begin with. For a given $v \in(0,1)$, define

$$
\Psi_{1}(N)=N^{v}, \quad N>0 .
$$

By Itô's formula, we have

$$
\begin{aligned}
\mathrm{d} \Psi_{1}(N) & \\
= & v N^{\nu-1} \mathrm{~d} N+0.5 v(v-1) N^{\nu-2}(\mathrm{~d} N)^{2} \\
= & v N^{v}\left[r(\kappa)+0.5(v-1) \varpi^{2}(\kappa)+\rho(\kappa) \frac{N^{a}(t)}{N^{a}(t)+\theta(\kappa)}-\beta(\kappa) N^{b}(t)\right] \mathrm{d} t \\
& +\nu \varpi(\kappa) N^{v} \mathrm{~d} \omega(t) \\
& \leq \nu N^{v}\left[r(\kappa)+\rho(\kappa) \frac{N^{a}(t)}{N^{a}(t)+\theta(\kappa)}-\beta(\kappa) N^{b}(t)\right] \mathrm{d} t+\nu \varpi(\kappa) N^{v} \mathrm{~d} \omega(t) .
\end{aligned}
$$

As a result,

$$
\begin{aligned}
& \mathrm{d}\left(e^{t} \Psi_{1}(N)\right) \\
& \quad=e^{t} \Psi_{1}(N) \mathrm{d} t+e^{t} \mathrm{~d} \Psi_{1}(N) \\
& \quad \leq v e^{t} N^{v}\left[1 / \nu+r(\kappa)+\rho(\kappa) \frac{N^{a}(t)}{N^{a}(t)+\theta}-\beta(\kappa) N^{b}(t)\right] \mathrm{d} t+e^{t} \nu \varpi(\kappa) N^{v} \mathrm{~d} \omega(t) \\
& \quad \leq v e^{t} N^{v}\left[1 / \nu+r^{u}+\rho^{u}-\beta^{l} N^{b}(t)\right] \mathrm{d} t+e^{t} \nu \varpi(\kappa) N^{v} \mathrm{~d} \omega(t) \\
& \quad \leq \Gamma e^{t} \mathrm{~d} t+e^{t} \nu \varpi(\kappa) N^{v} \mathrm{~d} \omega(t),
\end{aligned}
$$


here $\Gamma \in(0,+\infty)$ is a constant. Thereby,

$$
\limsup _{t \rightarrow+\infty} \mathbb{E}\left[N^{v}(t)\right] \leq \Gamma
$$

Let $\Lambda_{1}=(\Gamma / \varepsilon)^{1 / v}$, by Chebyshev's inequality [14],

$$
\limsup _{t \rightarrow+\infty} \mathrm{P}\left\{N(t)>\Lambda_{1}\right\}=\limsup _{t \rightarrow+\infty} \mathrm{P}\left\{N^{v}(t)>\Lambda_{1}^{v}\right\} \leq \limsup _{t \rightarrow+\infty} \mathbb{E}\left[N^{v}(t)\right] / \Lambda_{1}^{v}=\varepsilon
$$

Now we address (8). Let

$$
\Psi_{2}(N)=1 / N^{2}, \quad N>0 .
$$

Taking advantage of Itô’s formula yields

$$
\begin{aligned}
\mathrm{d} \Psi_{2}(N)= & -2 N^{-3} \mathrm{~d} N+3 N^{-4}(\mathrm{~d} N)^{2} \\
= & 2 \Psi_{2}(N)\left[\beta(\kappa) N^{b}(t)-\rho(\kappa) \frac{N^{a}(t)}{N^{a}(t)+\theta(\kappa)}-r(\kappa)\right] \mathrm{d} t \\
& +3 \varpi^{2}(\kappa) \Psi_{2}(N) \mathrm{d} t-2 \varpi(\kappa) \Psi_{2}(N) \mathrm{d} \omega(t) .
\end{aligned}
$$

From Lemma 1 we know that $M x=-2 \Delta+\bar{\Delta}(2, \ldots, 2)^{\mathrm{T}}$ possesses a solution which is represented as $\left(\xi_{1}, \ldots, \xi_{N^{*}}\right)^{\mathrm{T}}$, here $\Delta=\left(\Delta(1), \ldots, \Delta\left(N^{*}\right)\right)^{\mathrm{T}}$. Accordingly, for each $i \in \Upsilon$,

$$
\frac{1}{2} \sum_{l \in \Upsilon} m_{i l} \xi_{l}+\Delta(i)=\bar{\Delta}
$$

Fix a sufficiently small $\mu \in(0,1)$ such that, for all $i \in \Upsilon$,

$$
1-\xi_{i} \mu>0, \quad \bar{\Delta}-\mu \varpi^{2}(i)+\frac{\xi_{i} \mu}{2\left(1-\xi_{i} \mu\right)} \sum_{l \in \Upsilon} m_{i l} \xi_{l}>0 .
$$

It then follows from (9) that

$$
\begin{aligned}
\Delta(i)-\frac{1}{2\left(1-\xi_{i} \mu\right) \mu} \sum_{l \in \Upsilon} m_{i l}\left(1-\xi_{l} \mu\right) & =\Delta(i)+\frac{1}{2\left(1-\xi_{i} \mu\right)} \sum_{l \in \Upsilon} m_{i l} \xi_{l} \\
& =\bar{\Delta}+\frac{\xi_{i} \mu}{2\left(1-\xi_{i} \mu\right)} \sum_{l \in \Upsilon} m_{i l} \xi_{l} .
\end{aligned}
$$

Let

$$
\Psi_{3}(N, i)=\left(1-\xi_{i} \mu\right)\left(1+\Psi_{2}(N)\right)^{\mu}
$$

By Itô's formula,

$$
\mathbb{E} \Psi_{3}(N(t), \kappa(t))=\Psi_{3}(N(0), \kappa(0))+\mathbb{E} \int_{0}^{t} \mathcal{L} \Psi_{3}(N(s), \kappa(s)) \mathrm{d} s
$$


here

$\mathcal{L} \Psi_{3}(N, i)$

$$
\begin{aligned}
& =2\left(1-\xi_{i} \mu\right) \mu\left(1+\Psi_{2}(N)\right)^{\mu-2}\left\{\left(1+\Psi_{2}(N)\right)\right. \\
& \times\left[\Psi_{2}(N)\left(\beta(i) N^{b}-\rho(i) \frac{N^{a}}{N^{a}+\theta(i)}-r(i)+1.5 \varpi^{2}(i)\right)\right] \\
& \left.+(\mu-1) \varpi^{2}(i) \Psi_{2}^{2}(N)\right\}+\left(1+\Psi_{2}(N)\right)^{\mu} \sum_{l \in \Upsilon} m_{i l}\left(1-\xi_{l} \mu\right) \\
& =2\left(1-\xi_{i} \mu\right) \mu\left(1+\Psi_{2}(N)\right)^{\mu-2}\left\{-\left[\Delta(i)-\mu \varpi^{2}(i)\right] \Psi_{2}^{2}(N)\right. \\
& +\left(1.5 \varpi^{2}(i)-r(i)\right) \Psi_{2}(N)+\beta(i) N^{b} \Psi_{2}(N)\left(1+\Psi_{2}(N)\right) \\
& \left.-\rho(i) \frac{N^{a}}{N^{a}+\theta(i)} \Psi_{2}(N)\left(1+\Psi_{2}(N)\right)\right\}+\left(1+\Psi_{2}(N)\right)^{\mu} \sum_{l \in \Upsilon} m_{i l}\left(1-\xi_{l} \mu\right) \\
& =2\left(1-\xi_{i} \mu\right) \mu\left(1+\Psi_{2}(N)\right)^{\mu-2} \\
& \times\left\{-\left[\Delta(i)-\mu \varpi^{2}(i)-\frac{1}{2\left(1-\xi_{i} \mu\right) \mu} \sum_{l \in \Upsilon} m_{i l}\left(1-\xi_{l} \mu\right)\right] \Psi_{2}^{2}(N)\right. \\
& +\beta(i) \Psi_{2}^{2-b / 2}(N)+\left[1.5 \varpi^{2}(i)-r(i)+\frac{1}{\left(1-\xi_{i} \mu\right) \mu} \sum_{l \in \Upsilon} m_{i l}\left(1-\xi_{l} \mu\right)\right] \Psi_{2}(N) \\
& +\beta(i) \Psi_{2}^{1-b / 2}(N)+\frac{1}{2\left(1-\xi_{i} \mu\right) \mu} \sum_{l \in \Upsilon} m_{i l}\left(1-\xi_{l} \mu\right) \\
& \left.-\rho(i) \frac{N^{a}}{N^{a}+\theta(i)} \Psi_{2}(N)\left(1+\Psi_{2}(N)\right)\right\} \\
& =2\left(1-\xi_{i} \mu\right) \mu\left(1+\Psi_{2}(N)\right)^{\mu-2}\left\{-\left[\bar{\Delta}-\mu \varpi^{2}(i)+\frac{\xi_{i} \mu}{2\left(1-\xi_{i} \mu\right)} \sum_{l \in \Upsilon} m_{i l} \xi_{l}\right] \Psi_{2}^{2}(N)\right. \\
& +\beta(i) \Psi_{2}^{2-b / 2}(N)+\left[1.5 \varpi^{2}(i)-r(i)+\frac{1}{\left(1-\xi_{i} \mu\right) \mu} \sum_{l \in \Upsilon} m_{i l}\left(1-\xi_{l} \mu\right)\right] \Psi_{2}(N) \\
& +\beta(i) \Psi_{2}^{1-b / 2}(N)+\frac{1}{2\left(1-\xi_{i} \mu\right) \mu} \sum_{l \in \Upsilon} m_{i l}\left(1-\xi_{l} \mu\right) \\
& \left.-\rho(i) \frac{N^{a}}{N^{a}+\theta(i)} \Psi_{2}(N)\left(1+\Psi_{2}(N)\right)\right\} \text {. }
\end{aligned}
$$

One can verify that

$$
\begin{aligned}
(1 & \left.+\Psi_{2}(N)\right)^{\mu} \sum_{l \in \Upsilon} m_{i l}\left(1-\xi_{l} \mu\right) \\
& =\left[2\left(1-\xi_{i} \mu\right) \mu\left(1+\Psi_{2}(N)\right)^{\mu-2}\right]\left[\left(1+\Psi_{2}(N)\right)^{2} \frac{\sum_{l \in \Upsilon} m_{i l}\left(1-\xi_{l} \mu\right)}{2\left(1-\xi_{i} \mu\right) \mu}\right] \\
& =\left[2\left(1-\xi_{i} \mu\right) \mu\left(1+\Psi_{2}(N)\right)^{\mu-2}\right]\left[\frac{\sum_{l \in \Upsilon} m_{i l}\left(1-\xi_{l} \mu\right)}{2\left(1-\xi_{i} \mu\right) \mu} \Psi_{2}^{2}(N)\right.
\end{aligned}
$$




$$
\left.+\frac{\sum_{l \in \Upsilon} m_{i l}\left(1-\xi_{l} \mu\right)}{\left(1-\xi_{i} \mu\right) \mu} \Psi_{2}(N)+\frac{\sum_{l \in \Upsilon} m_{i l}\left(1-\xi_{l} \mu\right)}{2\left(1-\xi_{i} \mu\right) \mu}\right]
$$

Fix a sufficiently small $\gamma>0$ such that, for all $i \in \Upsilon$,

$$
\bar{\Delta}-\mu \varpi^{2}(i)+\frac{\xi_{i} \mu}{2\left(1-\xi_{i} \mu\right)} \sum_{l \in \Upsilon} m_{i l} \xi_{l}-\frac{\gamma}{2 \mu}>0 .
$$

Let

$$
\Psi_{4}(N, i)=e^{\gamma t} \Psi_{3}(N, i)
$$

By Itô’s formula,

$$
\mathbb{E} \Psi_{4}(N(t), \mu(t))=\Psi_{3}(N(0), \mu(0))+\mathbb{E} \int_{0}^{t} \mathcal{L}\left[e^{\gamma s} \Psi_{3}(N(s), \mu(s))\right] \mathrm{d} s,
$$

here

$$
\begin{aligned}
\mathcal{L}\left[\Psi_{4}(N, i)\right] & \\
= & e^{\gamma t} \mathcal{L} \Psi_{3}(N, i)+\gamma e^{\gamma t} \Psi_{3}(N, i) \\
= & e^{\gamma t} 2\left(1-\xi_{i} \mu\right) \mu\left(1+\Psi_{2}(N)\right)^{\mu-2} \\
& \times\left\{-\left[\bar{\Delta}-\mu \varpi^{2}(i)-\frac{\gamma}{2 \mu}+\frac{\xi_{i} \mu}{2\left(1-\xi_{i} \mu\right)} \sum_{l \in \Upsilon} m_{i l} \xi_{l}\right] \Psi_{2}^{2}(N)\right. \\
& +\beta(i) \Psi_{2}^{2-b / 2}(N)+\left[1.5 \varpi^{2}(i)-r(i)+\frac{1}{\left(1-\xi_{i} \mu\right) \mu} \sum_{l \in \Upsilon} m_{i l}\left(1-\xi_{l} \mu\right)+\frac{\gamma}{\mu}\right] \Psi_{2}(N) \\
& +\beta(i) \Psi_{2}^{1-b / 2}(N)+\frac{1}{2\left(1-\xi_{i} \mu\right) \mu} \sum_{l \in \Upsilon} m_{i l}\left(1-\xi_{l} \mu\right)+\frac{\gamma}{2 \mu} \\
& \left.-\rho(i) \frac{N^{a}}{N^{a}+\theta(i)} \Psi_{2}(N)\left(1+\Psi_{2}(N)\right)\right\} \\
\leq & e^{\gamma t} 2\left(1-\xi_{i} \mu\right) \mu\left(1+\Psi_{2}(N)\right)^{\mu-2} \\
& \times\left\{-\left[\bar{\Delta}-\mu \beta_{1}^{2}(i)-\frac{\gamma}{2 \mu}+\frac{\xi_{i} \mu}{2\left(1-\xi_{i} \mu\right)} \sum_{l \in \Upsilon} m_{i l} \xi_{l}\right] \Psi_{2}^{2}(N)\right. \\
& +\beta(i) \Psi_{2}^{2-b / 2}(N)+\left[1.5 \varpi^{2}(i)-r(i)+\frac{1}{\left(1-\xi_{i} \mu\right) \mu} \sum_{l \in \Upsilon} m_{i l}\left(1-\xi_{l} \mu\right)+\frac{\gamma}{\mu}\right] \Psi_{2}(N) \\
& \left.+\beta(i) \Psi_{2}^{1-b / 2}(N)+\frac{1}{2\left(1-\xi_{i} \mu\right) \mu} \sum_{l \in \Upsilon} m_{i l}\left(1-\xi_{l} \mu\right)+\frac{\gamma}{2 \mu}\right\} \\
=: & e^{\gamma t}\left(1-\xi_{i} \mu\right) \Gamma(N, i)
\end{aligned}
$$

and

$\Gamma(N, i)$

$$
=2 \mu\left(1+\Psi_{2}(N)\right)^{\mu-2}\left\{-\left[\bar{\Delta}-\mu \beta_{1}^{2}(i)-\frac{\gamma}{2 \mu}+\frac{\xi_{i} \mu}{2\left(1-\xi_{i} \mu\right)} \sum_{l \in \Upsilon} m_{i l} \xi_{l}\right] \Psi_{2}^{2}(N)\right.
$$




$$
\begin{aligned}
& +\beta(i) \Psi_{2}^{2-b / 2}(N)+\left[1.5 \varpi^{2}(i)-r(i)+\frac{1}{\left(1-\xi_{i} \mu\right) \mu} \sum_{l \in \Upsilon} m_{i l}\left(1-\xi_{l} \mu\right)+\frac{\gamma}{\mu}\right] \Psi_{2}(N) \\
& \left.+\beta(i) \Psi_{2}^{1-b / 2}(N)+\frac{1}{2\left(1-\xi_{i} \mu\right) \mu} \sum_{l \in \Upsilon} m_{i l}\left(1-\xi_{l} \mu\right)+\frac{\gamma}{2 \mu}\right\} .
\end{aligned}
$$

By (11),

$$
\tilde{\Gamma}_{1}:=\sup _{N>0, i \in \Upsilon} \Gamma(N, i)<+\infty .
$$

Hence

$$
\left(1-\xi_{i} \mu\right) \mathbb{E}\left[e^{\gamma t}\left(1+\Psi_{2}(N(t))\right)^{\mu}\right] \leq\left(1-\xi_{i} \mu\right)\left(1+\Psi_{2}(N(0))\right)^{\mu}+\left(1-\xi_{i} \mu\right) \tilde{\Gamma}_{1}\left(e^{\gamma t}-1\right) / \gamma
$$

Therefore,

$$
\limsup _{t \rightarrow+\infty} \mathbb{E}\left[\Psi_{2}^{\mu}(N(t))\right] \leq \limsup _{t \rightarrow+\infty} \mathbb{E}\left[\left(1+\Psi_{2}(N(t))\right)^{\mu}\right] \leq \tilde{\Gamma}_{1} / \theta=: \tilde{\Gamma}_{2}
$$

That is to say

$$
\limsup _{t \rightarrow+\infty} \mathbb{E}\left[N^{-2 \mu}(t)\right] \leq \tilde{\Gamma}_{2}
$$

Let $\Lambda_{2}=\left(\varepsilon / \tilde{\Gamma}_{2}\right)^{0.5 / \mu}$. Using the Chebyshev inequality,

$$
\mathrm{P}\left\{N(t)<\Lambda_{2}\right\}=\mathrm{P}\left\{N^{-2 \mu}(t)>\Lambda_{2}^{-2 \mu}\right\} \leq \mathbb{E}\left[N^{-2 \mu}(t)\right] / \Lambda_{2}^{-2 \mu}=\Lambda_{2}^{2 \mu} \mathbb{E}\left[N^{-2 \mu}(t)\right]
$$

Hence,

$$
\limsup _{t \rightarrow+\infty} P\left\{N(t)<\Lambda_{2}\right\} \leq \Lambda_{2}^{2 \mu} \tilde{\Gamma}_{2}=\varepsilon
$$

So

$$
\liminf _{t \rightarrow+\infty} \mathrm{P}\left\{N(t) \geq \Lambda_{2}\right\} \geq 1-\varepsilon
$$

\section{Stability}

In the investigation of stochastic population models, the existence and uniqueness of an invariant measure are key topics [18]. Now let us give a sufficient condition for the existence and uniqueness of an invariant measure of model (3).

Theorem 3 If $\bar{\Delta}>0$, then model (3) has a unique invariant measure which is concentrated on $(0,+\infty) \times \Upsilon$.

Proof Fix a sufficiently small $\sigma>0$ such that

$$
1-\frac{\sigma}{2} \xi^{u}>0, \quad \bar{\Delta}-\frac{\sigma}{2}\left(\varpi^{2}\right)^{u}+\frac{\sigma}{2} \min _{i \in \Upsilon}\left\{\frac{\xi_{i}(\bar{\Delta}-\Delta(i))}{1-\sigma \xi_{i} / 2}\right\}>0,
$$


where $\xi_{i}$ satisfies (9). Let

$$
\Psi_{5}(N, i)=\left(1-\frac{\sigma \xi_{i}}{2}\right) N^{-\sigma}+N, \quad N>0
$$

Then

$$
\begin{aligned}
& \mathcal{L} \Psi_{5}(N, i) \\
& =-\sigma\left(1-\frac{\sigma \xi_{i}}{2}\right) N^{-\sigma}\left(r(i)+\rho(i) \frac{N^{a}}{N^{a}+\theta(i)}-\beta(i) N^{b}\right) \\
& +\frac{\varpi^{2}(i)}{2} \sigma(\sigma+1)\left(1-\frac{\sigma \xi_{i}}{2}\right) N^{-\sigma} \\
& -\sigma N^{-\sigma} \sum_{l \in \Upsilon} m_{i l} \frac{\xi_{l}}{2}+N\left(r(i)+\rho(i) \frac{N^{a}}{N^{a}+\theta(i)}-\beta(i) N^{b}\right) \\
& =-\sigma\left(1-\frac{\sigma \xi_{i}}{2}\right) N^{-\sigma}\left(r(i)-\frac{1}{2} \varpi^{2}(i)-\frac{\sigma}{2} \varpi^{2}(i)\right) \\
& -\sigma\left(1-\frac{\sigma \xi_{i}}{2}\right) N^{-\sigma}\left(1+\frac{\sigma \xi_{i} / 2}{1-\sigma \xi_{i} / 2}\right) \sum_{l \in \Upsilon} m_{i l} \frac{\xi_{l}}{2} \\
& -\sigma\left(1-\frac{\sigma \xi_{i}}{2}\right) N^{-\sigma}\left(\rho(i) \frac{N^{a}}{N^{a}+\theta(i)}-\beta(i) N^{b}\right) \\
& \left.+N\left(r(i)+\rho(i) \frac{N^{a}}{N^{a}+\theta(i)}-\beta(i) N^{b}\right)\right) \\
& =-\sigma\left(1-\frac{\sigma \xi_{i}}{2}\right) N^{-\sigma}\left(\Delta(i)+\frac{1}{2} \sum_{l \in \Upsilon} m_{i l} \xi_{l}-\frac{\sigma}{2} \varpi^{2}(i)+\frac{\sigma \xi_{i} / 2}{1-\sigma \xi_{i} / 2} \sum_{l \in \Upsilon} m_{i l} \frac{\xi_{l}}{2}\right) \\
& -\sigma\left(1-\frac{\sigma \xi_{i}}{2}\right) N^{-\sigma}\left(\rho(i) \frac{N^{a}}{N^{a}+\theta(i)}-\beta(i) N^{b}\right) \\
& \left.+N\left(r(i)+\rho(i) \frac{N^{a}}{N^{a}+\theta(i)}-\beta(i) N^{b}\right)\right) \\
& =-\sigma\left(1-\frac{\sigma \xi_{i}}{2}\right) N^{-\sigma}\left(\bar{\Delta}-\frac{\sigma}{2} \varpi^{2}(i)+\frac{\sigma \xi_{i} / 2}{1-\sigma \xi_{i} / 2}(\bar{\Delta}-\Delta(i))\right) \\
& -\sigma\left(1-\frac{\sigma \xi_{i}}{2}\right) N^{-\sigma}\left(\rho(i) \frac{N^{a}}{N^{a}+\theta(i)}-\beta(i) N^{b}\right) \\
& \left.+N\left(r(i)+\rho(i) \frac{N^{a}}{N^{a}+\theta(i)}-\beta(i) N^{b}\right)\right) \text {. }
\end{aligned}
$$

It then follows from (12) that

$$
\lim _{N \rightarrow 0} \frac{\mathcal{L} \Psi_{5}(N, i)}{-\sigma\left(1-\sigma \xi_{i} / 2\right) N^{-\sigma}\left(\bar{\Delta}-\frac{\sigma}{2} \varpi^{2}(i)+\frac{\sigma \xi_{i} / 2}{1-\sigma \xi_{i} / 2}(\bar{\Delta}-\Delta(i))\right)}=1
$$

and

$$
\lim _{N \rightarrow+\infty} \frac{\mathcal{L} \Psi_{5}(N, i)}{-\beta(i) N^{1+b}}=1
$$


By (13) and (14), there is a $a_{1} \in(0,1)$ such that, for $N \leq a_{1}$ or $N \geq 1 / a_{1}$,

$$
\mathcal{L} \Psi_{5}(N, i) \leq-1
$$

Let $U=\left(a_{1}, 1 / a_{1}\right)$, we have, for any $(N, i) \in\{(0,+\infty) \backslash U\} \times \Upsilon$,

$$
\mathcal{L} \Psi_{5}(N, i) \leq-1
$$

Then the desired assertion follows from Theorem 3.13 and Theorem 4.3 in [21].

\section{Discussions and simulations}

In this section, let us see the impacts of environmental noises on the extermination, persistence and invariant measure of model (3).

Based on Theorems 1-3, if $\phi$, the distribution of $\kappa(t)$, makes $\bar{\Delta}>0$, then the hybrid system (3) is persistent, and it has a unique invariant measure on $(0,+\infty) \times \Upsilon$; if $\phi$ makes $\bar{\Delta}+\bar{\rho}<0$, then the species represented by (3) vanishes. To see these functions more directly, let $N^{*}=2$, then (3) has the two subsystems

$$
\mathrm{d} N(t)=N(t)\left[r(1)+\frac{\rho(1) N^{a}(t)}{N^{a}(t)+\theta(1)}-\beta(1) N^{b}(t)\right] \mathrm{d} t+\varpi(1) N(t) \mathrm{d} \omega(t)
$$

and

$$
\mathrm{d} N(t)=N(t)\left[r(2)+\frac{\rho(2) N^{a}(t)}{N^{a}(t)+\theta(2)}-\beta(2) N^{b}(t)\right] \mathrm{d} t+\varpi(2) N(t) \mathrm{d} \omega(t) .
$$

Suppose that in state 1 and state $2, r(1)-\varpi^{2}(1) / 2+\rho(1)<0$ and $r(2)-\varpi^{2}(2) / 2>0$, respectively. Then the species represented by subsystem (15) vanishes (see Fig. 1); subsystem (16) is persistent, and it owns a unique invariant measure on $(0,+\infty)$ (see Fig. 2). If $\phi$ makes $\sum_{i=1}^{2} \phi_{i}\left[r(i)-\varpi^{2}(i) / 2+\rho(i)\right]<0$, then the species represented by (3) vanishes (see Fig. 3); If $\phi$ makes $\sum_{i=1}^{2} \phi_{i}\left[r(i)-\varpi^{2}(i) / 2\right]>0$, then (3) is persistent, and it owns a unique invariant measure on $(0,+\infty) \times\{1,2\}$ (see Fig. 4 ).

Now we probe the functions of white noise. According to Theorem 1, if $\bar{\Delta}+\bar{\rho}=$ $\sum_{i \in \Upsilon} \phi_{i}\left[r(i)+\rho(i)-\varpi^{2}(i) / 2\right]<0$, then the species vanishes. Hence, sufficiently large white noise on the growth rate could lead to the extermination of the species, which is in accord with the classical results [3]. To observe this more clearly, let see Fig. 5 where the parameter values are the same as those in Fig. 4 except $\varpi^{2}(2)=0.8$. Figure 4 shows that the species represented by (3) is persistent when $\varpi^{2}(2)=0.2$. However, when the value of $\varpi^{2}(2)$ changes to 0.8 , Fig. 5 shows that the species represented by (3) vanishes.

\section{Conclusions}

In the field of mathematical biology, it is meaningful to probe the functions of the environmental noises on the features of population models [11]. In the present research, we have developed a stochastic differential equation population model with Allee effects. We have analyzed the extermination, persistence and invariant measure of the model.

The findings uncover that the features of the model are closely related to the environmental noises. First, if the Markov chain $\kappa(t)$ spends more time on the desired states 


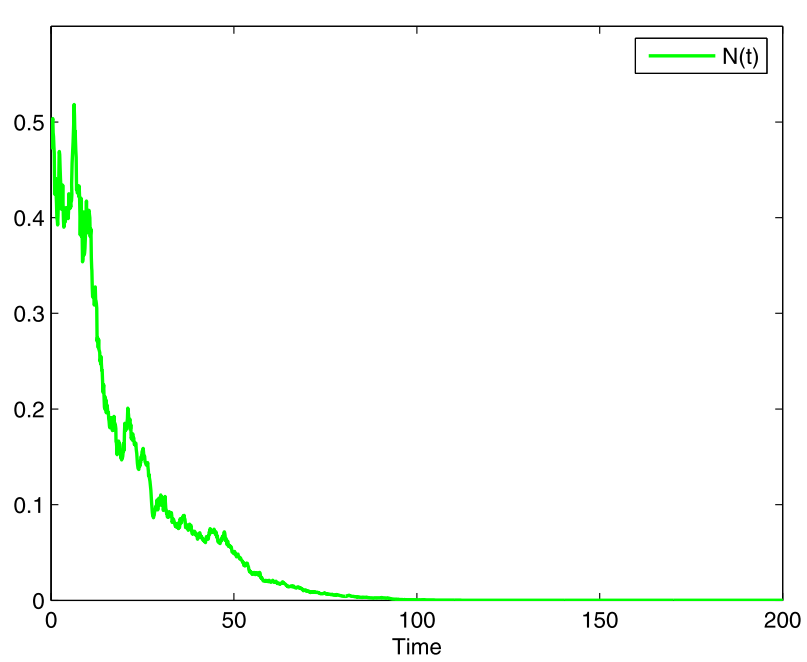

Figure 1 A trajectory of subsystem (15) with $r(1)=0.2, \rho(1)=0.1, \varpi^{2}(1)=0.8, a=1, \theta(1)=0.1, \beta(1)=0.05$, $b=2$. This figure suggests that the species represented by subsystem (15) vanishes
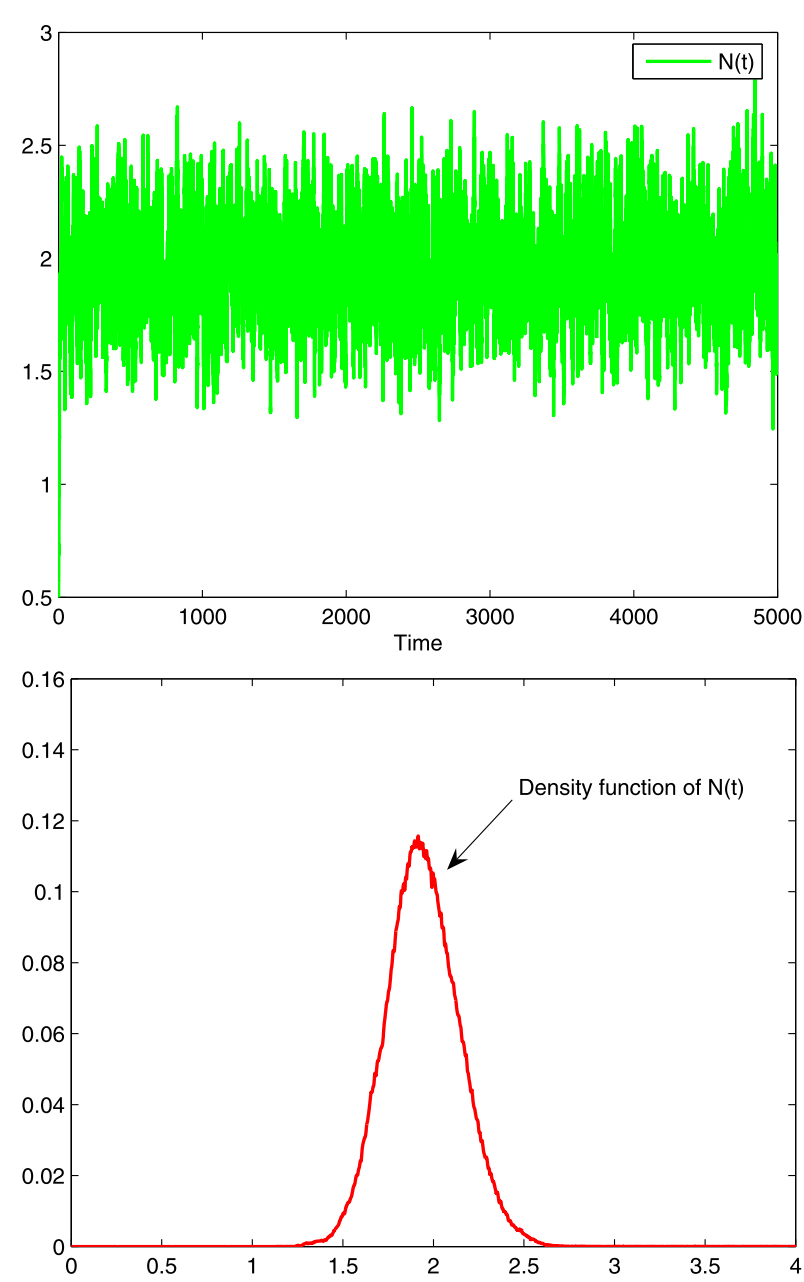

Figure 2 Subsystem (16) with $r(2)=0.2, \rho(2)=0.1, \varpi^{2}(2)=0.2, a=1, \theta(2)=0.1, \beta(2)=0.05, b=2$. (a) is a trajectory of the system which suggests that the system is persistent; (b) is the density function of the solution of the system $t=3000$ 


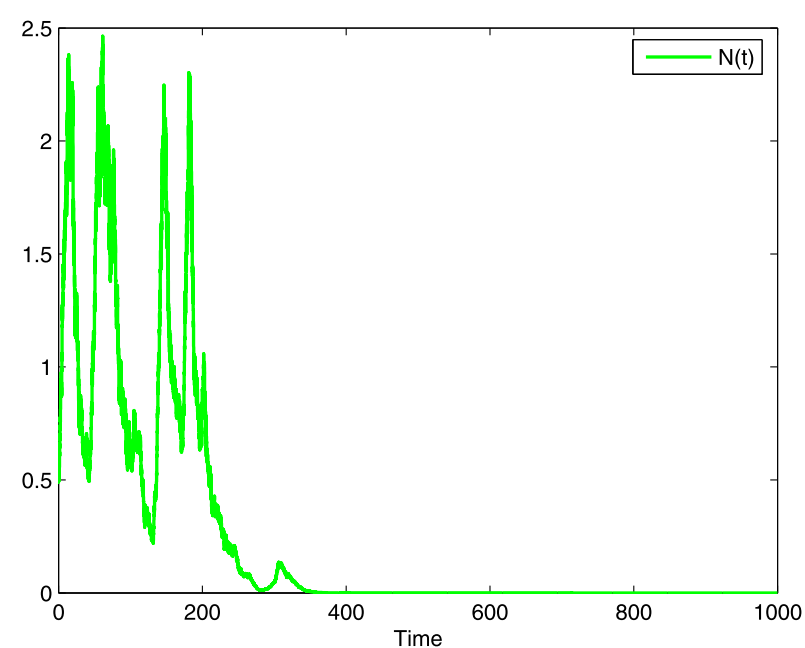

Figure 3 A trajectory of hybrid system (3) with parameter values given in Fig. 1 and Fig. 2, and $\phi=(0.8,0.2)$. This figure suggests that the species represented by hybrid system (3) vanishes
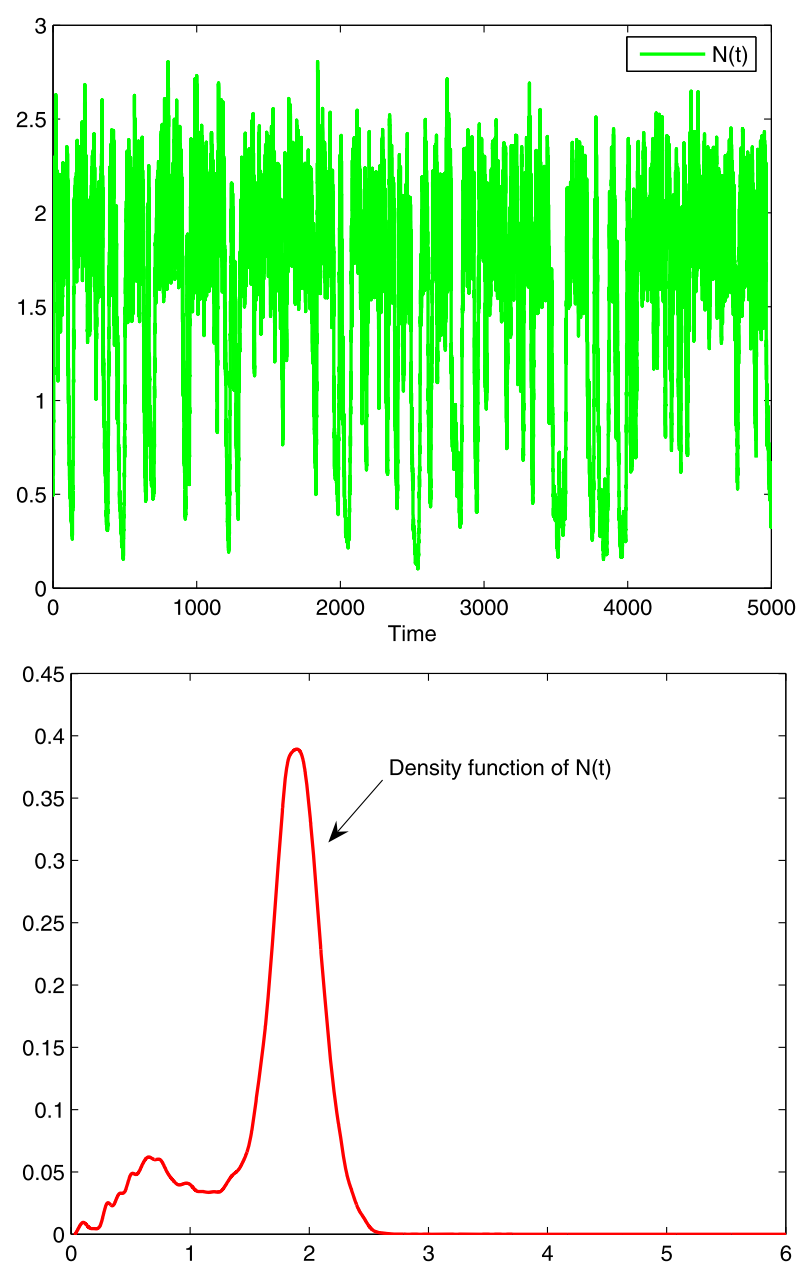

Figure 4 Hybrid system (16) with parameter values given in Fig. 1 and Fig. 2 , and $\phi=(0.2,0.8)$. (a) is a trajectory of the system which suggests that the system is persistent; $(\mathbf{b})$ is the density function of the solution of the system $t=3000$ 


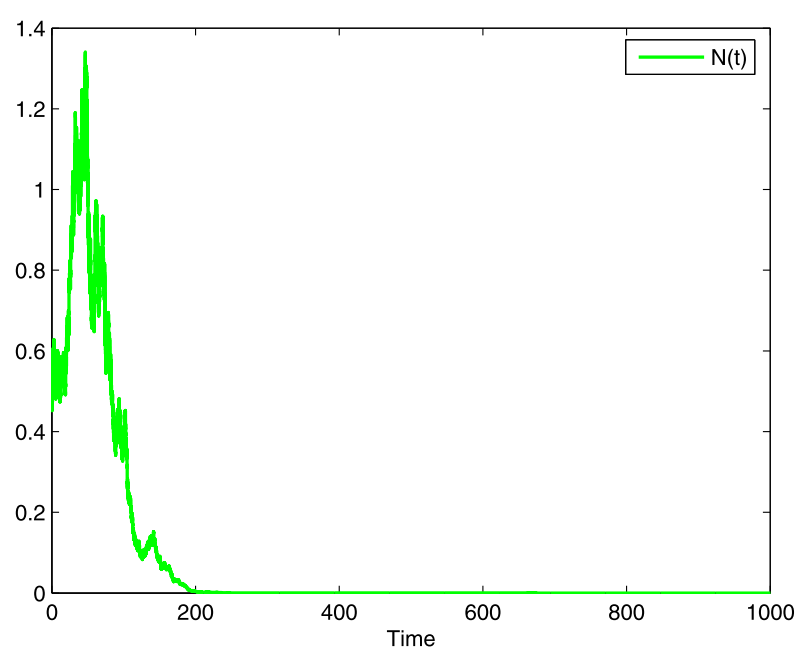

Figure 5 Hybrid system (16) with parameter values given in Fig. 4 except $\varpi^{2}(2)=0.8$. This figure suggests that the species represented by system (16) vanishes

(the states where $r(\cdot)-\varpi^{2}(\cdot) / 2>0$ ), then the species is persistent (see Fig. 4); conversely, if the Markov chain $\kappa(t)$ spends more time on the undesired states (the states where $r(\cdot)+\rho(\cdot)-\varpi^{2}(\cdot) / 2<0$ ), then the species vanishes (see Fig. 3). Second, sufficiently large white noise on the growth rate could lead to the extermination of the species (compare Fig. 4 with Fig. 5).

Some issues are not analyzed in this research. First, the case that $\bar{\Delta}+\bar{\rho}>0>\bar{\Delta}$ is not settled yet. Second, model (3) presumes that white noise only affects $r$; the case that other parameters are also affected by white noise is not settled yet. Finally, there are some sudden environmental noises which can be described by Lévy jumps (see, e.g., [1]). It is interesting to consider model (3) with Lévy jumps.

\section{Acknowledgements}

The authors thank the editor and the referees for their careful reading and valuable comments.

Funding

Guixin Hu is supported by the Fundamental Research Funds for the Universities of Henan Province (NSFRF200321).

Availability of data and materials

All data generated or analysed during this study are included in this published article.

Competing interests

The authors declare that they have no competing interests.

\section{Authors' contributions}

FMZ finished the establishment of model and development, GXH mainly finished the writing of the whole content of the paper. Both authors read and approved the final manuscript.

\section{Author details}

${ }^{1}$ School of Mathematical Science, Huaiyin Normal University, Huaian, 223300, P.R. China. ${ }^{2}$ School of Mathematics and Information Science, Henan Polytechnic University, Jiaozuo, 454000, P.R. China.

\section{Publisher's Note}

Springer Nature remains neutral with regard to jurisdictional claims in published maps and institutional affiliations. 


\section{References}

1. Bao, J., Mao, X., Yin, G., Yuan, C.: Competitive Lotka-Volterra population dynamics with jumps. Nonlinear Anal. 74, $6601-6616(2011)$

2. Braumann, C.: Variable effort harvesting models in random environments: generalization to density-dependent noise intensities. Math. Biosci. 177/178, 229-245 (2002)

3. Chesson, P.L.: The stabilizing effect of a random environment. J. Math. Biol. 15, 1-36 (1982)

4. Gopalsamy, K., Ladas, G.: On the oscillation and asymptotic behavior of $\dot{N}(t)=N(t)\left[a+b N(t-\tau)-c N^{2}(t-\tau)\right]$. Q. Appl. Math. 48, 433-440 (1990)

5. Jacobs, J.: Cooperation, optimal density and low density thresholds: yet another modification of the logistic model. Oecologia 64, 389-395 (1984)

6. Ji, W., Zhang, Y., Liu, M.: Dynamical bifurcation and explicit stationary density of a stochastic population model with Allee effects. Appl. Math. Lett. 111, 106662 (2021)

7. Jovanović, M., Krstić, M.: The influence of time-dependent delay on behavior of stochastic population model with the Allee effect. Appl. Math. Model. 39, 733-746 (2015)

8. Kang, Y., Udiani, O.: Dynamics of a single species evolutionary model with Allee effects. J. Math. Anal. Appl. 418 492-515 (2014)

9. Khasminskii, R., Zhu, C., Yin, G.: Stability of regime-switching diffusions. Stoch. Process. Appl. 117, 1037-1051 (2007)

10. Lande, R., Engen, S., Saether, B.: Stochastic Population Dynamics in Ecology and Conservation. Oxford University Press, Demand (2003)

11. Liu, M., Bai, C.: Analysis of a stochastic tri-trophic food-chain model with harvesting. J. Math. Biol. 73, 597-625 (2016)

12. Liu, M., Deng, M.: Analysis of a stochastic hybrid population model with Allee effect. Appl. Math. Comput. 364, $124582(2020)$

13. Liu, M., Wang, K.: Stochastic Lotka-Volterra systems with Lévy noise. J. Math. Anal. Appl. 410, 750-763 (2014)

14. Mao, X., Yuan, C.: Stochastic Differential Equations with Markovian Switching. Imperial College Press, London (2006)

15. May, R.: Stability and Complexity in Model Ecosystems. Princeton Univ. Press, New York (1973)

16. Molles, J., Manuel, C.: Ecology: Concepts and Applications, 4th edn. McGraw-Hill, New York (2008)

17. Odum, E., Barrett, G.: Fundamentals of Ecology, 5th edn. Cengage Learning, Singapore (1980)

18. Wang, K.: Stochastic Mathematical Biology Models. Science Press, Beijing (2010)

19. Wu, R., Zou, X., Wang, K.: Asymptotic properties of stochastic hybrid Gilpin-Ayala system with jumps. Appl. Math. Comput. 249, 53-66 (2014)

20. Yu, X., Yuan, S., Zhang, T.: Persistence and ergodicity of a stochastic single species model with Allee effect under regime switching. Commun. Nonlinear Sci. Numer. Simul. 59, 359-374 (2018)

21. Zhu, C., Yin, G.: Asymptotic properties of hybrid diffusion systems. SIAM J. Control Optim. 46, 1155-1179 (2007)

\section{Submit your manuscript to a SpringerOpen ${ }^{\circ}$ journal and benefit from:}

- Convenient online submission

- Rigorous peer review

- Open access: articles freely available online

- High visibility within the field

- Retaining the copyright to your article

Submit your next manuscript at $>$ springeropen.com 